\title{
Confirmation of Age-dependence in the Leakage of Contrast Medium around the Cortical Veins into Cerebrospinal Fluid after Intravenous Administration of Gadolinium-based Contrast Agent
}

\author{
Shinji Naganawa ${ }^{1 *}$, Rintaro Ito ${ }^{1}$, Hisashi Kawai ${ }^{1}$, Toshiaki Taoka ${ }^{1}$, \\ Tadao Yoshida ${ }^{2}$, and Michihiko Sone ${ }^{2}$
}

\begin{abstract}
Purpose: It has been reported previously that intravenously administered gadolinium-based contrast agent (GBCA) leaks into the subarachnoid space around the cortical veins at $4 \mathrm{~h}$ after injection in all old people over 37 years, but not in younger people up to 37 years of age in 3D-real IR images. The purpose of this study was to investigate whether there was a strict threshold of 37 years of age for the leakage of the GBCA into the subarachnoid space.
\end{abstract}

Methods: The subjects included 190 patients, that were scanned for 3D-real IR images at 4 hours after intravenous injection of GBCA as a diagnostic test for endolymphatic hydrops. The patient's age ranged from 14 to 81 years. Two experienced neuroradiologists evaluated the images to determine whether the GBCA leakage around the cortical veins was positive or negative. Any discrepancies between the two observers were discussed and a consensus was obtained.

A Mann-Whitney $U$ test and receiver operating characteristic (ROC) curve analysis were used to compare the positive and the negative group and to set the age cut-off value for the prediction of GBCA leakage.

Results: The GBCA leakage around the cortical veins was negative in 35 patients and positive in 155 patients. The average age was $33 \pm 11$ years in the negative group, and $55 \pm 12$ years in the positive group $(P<0.01)$. In the ROC analysis for the age and leakage of the GBCA, an area under the curve was 0.905 and the cut-off age was 37.317 years (sensitivity of 0.942 and specificity of 0.771 ).

Conclusion: Intravenously administered GBCA leaks into the subarachnoid space around the cortical veins in most patients over 37 years of age. However, it should be noted that it can be found occasionally in patients under 37 years of age.

Keywords: magnetic resonance imaging, cortical vein, gadolinium, glymphatic system

\section{Introduction}

It has been reported previously that gadolinium-based contrast agents (GBCAs) leak into the subarachnoid space around the cortical veins on the brain surface at $4 \mathrm{~h}$ after intravenous injection in 3D-real IR images. ${ }^{1}$ This 3D-real IR technique is

${ }^{1}$ Department of Radiology, Nagoya University Graduate School of Medicine, Aichi, Japan

${ }^{2}$ Department of Otorhinolaryngology, Nagoya University Graduate School of Medicine, Aichi, Japan

${ }^{*}$ Corresponding author: Department of Radiology, Nagoya University Graduate School of Medicine, 65 Tsurumai-cho, Shouwa-ku, Nagoya, Aichi 4668550, Japan. Phone: +81-52-744-2327, Fax: +81-52-744-2335, E-mail: naganawa@med.nagoya-u.ac.jp

(C)2020 Japanese Society for Magnetic Resonance in Medicine

This work is licensed under a Creative Commons Attribution-NonCommercialNoDerivatives International License.

Received: November 25, 2019 | Accepted: January 3, 2020 modified from heavily $\mathrm{T}_{2}$-weighted $3 \mathrm{D}$-FLAIR, and is quite sensitive to low concentrations of GBCA in fluid..$^{2-7}$ In a previous study, it was reported that no leakage of GBCA into the subarachnoid space was detected in young people up to 37 years of age on 3D-real IR imaging. ${ }^{1}$ However, that study included only 21 cases total, and only five people under the age of $37 .{ }^{1}$

The finding that GBCA leaks into the subarachnoid space around the cortical veins might be related to the function of the waste clearance system from the brain (i.e.: the glymphatic system or the meningeal lymphatic channels). ${ }^{1,8-12}$ The concepts of the glymphatic system ${ }^{13-15}$ and the meningeal lymphatic channels ${ }^{9,10}$ are currently attracting much attention from the neuroscience community.

The first objective of this current study was to investigate whether there was a strict threshold of 37 years of age for the leakage of the GBCA into the subarachnoid space after intravenous injection as was reported previously. Since 
there is a hypothesis that GBCA leaks around the cortical veins in part due to the influence of arachnoid granulations protruding into the venous sinus, ${ }^{16}$ the number of protruding granulations into the superior sagittal sinus (SSS) might be important. The secondary purpose of this study was to investigate the relationship between the number of arachnoid granulations protruding into the SSS and the leakage of the GBCA into the subarachnoid space around the cortical veins.

\section{Materials and Methods}

\section{Patients}

The subjects included 190 patients (91 male and 99 female), that were scanned at $4 \mathrm{~h}$ after an intravenous injection of gadolinium as a diagnostic test for endolymphatic hydrops. The patient age ranged from 14 to 81 years and the median age was 50 years. For the age calculation, the value obtained by dividing the number of days from the date of birth to the date of the examination by 365.25 was used. In all cases, the estimated glomerular filtration rate (eGFR) was $60 \mathrm{~mL} / \mathrm{min} /$ $1.73 \mathrm{~m}^{2}$ or greater. There were no cases with brain tumors or large cerebral infarctions. They do not have apparent history of central nervous system infection. Systemic steroids had not been administered within 2 months prior to the MR examination. In the 190 patients, 21 patients evaluated in the previous study ${ }^{1}$ were included. Images from these 21 patients were evaluated again in the present study.

\section{MR imaging}

The axial 3D-real IR images covering the entire skull were obtained at $4 \mathrm{~h}$ after an intravenous injection of a single dose $(0.1 \mathrm{mmol} / \mathrm{kg})$ of macrocyclic GBCA (Gadobutrol, Bayerpharm, Osaka, Japan). A 3T MR scanner (Skyra, Siemens Healthineers, Erlangen, Germany) with a 32-channel array coil was used. The detailed parameters for the 3D-real IR imaging were the same as the previous study ${ }^{1}$ and are indicated in Table 1.

\section{Image analysis}

A high signal intensity in the subarachnoid space around the cortical veins with a value of 30 or greater, a length of at least $10 \mathrm{~mm}$ and a width of at least $2 \mathrm{~mm}$ on the 3D-real IR images was regarded as a positive finding. Therefore, the thin pial high signal intensity on the brain surface, the pial-sheath around the cortical veins and the presumed meningeal lymphatics along both sides of the superior sagittal sinus were excluded (Figs. 1 and 2). On a PACS viewer (RapideyeCore, Canon Medical Systems, Tokyo, Japan), the 3D-real IR images were displayed with very narrowed window conditions (Window width of 2, Window level of 30), as well as regular window conditions (Window width of 80, Window level of 10), which showed the anatomy of the brain, subarachnoid spaces and cortical veins (Figs. 1 and 2). The regular window parameters are identical to those in the previous study. ${ }^{1}$ Two experienced neuroradiologists (S.N. and T.T.)

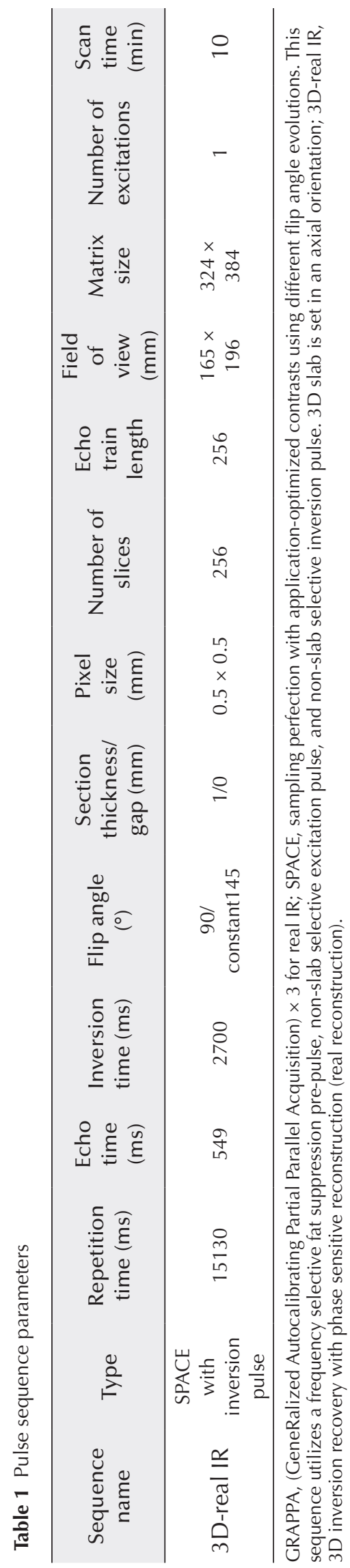



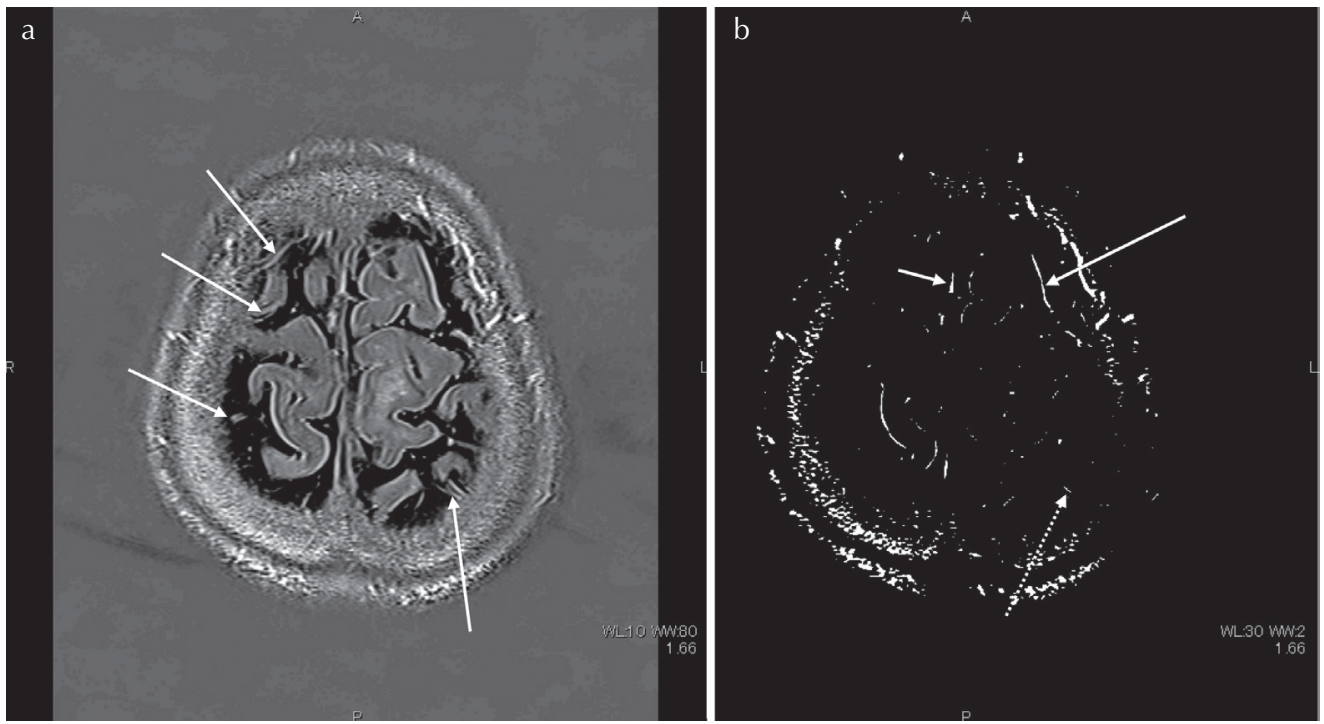

Fig. 1 A 32-year-old man with a suspicion of endolymphatic hydrops. The left image is the regular window width and level setting (window width of 80 and window level of 10). The right image is the extremely narrow window width setting (window width of 2 and window level of 30) at the same location as the left image. The pial-sheath around the cortical vein shows a linear high signal intensity (arrows, a). In the right image, the thin pial high signal intensity on the brain surface (arrow, b), the pial-sheath around the cortical veins (dotted arrow, b), and the presumed meningeal lymphatics along the superior sagittal sinus (short arrow, b) can be seen as thin structures with a high signal intensity. However, these structures do not match the criteria for a positive finding. Thus, we regarded this image as negative.
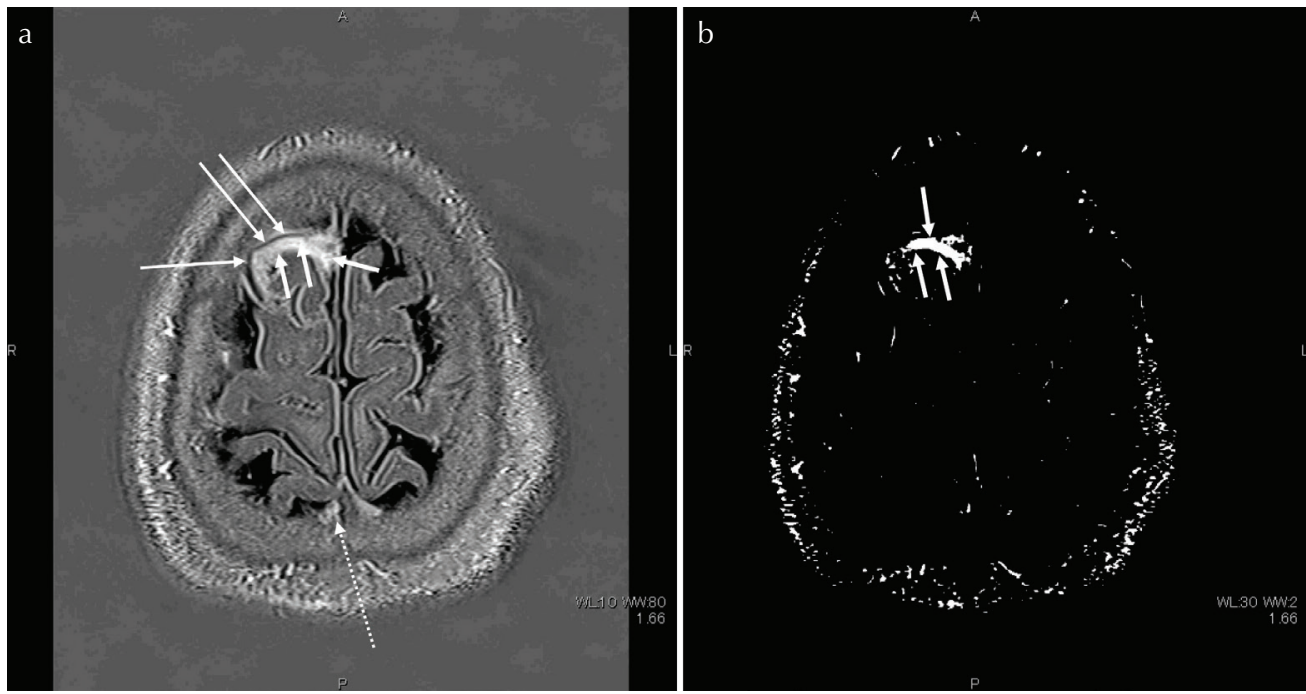

Fig. 2 A 31-year-old man with a suspicion of endolymphatic hydrops. The left image is the regular window width and level setting (window width of 80 and window level of 10). The right image is the extremely narrow window width setting (window width of 2 and window level of 30) at the same location as the left image. Around the cortical vein (arrows, a), the leakage of gadolinium-based contrast agent is indicated as a band-like high signal intensity (short arrows, a). An arachnoid granulation is protruding into the superior sagittal sinus (dotted arrow, a). In the right image, the leakage of the gadolinium-based contrast agent is indicated as a band-like high signal intensity (short arrow, b). This broad-shaped structure matches the criteria for a positive finding. Thus, we regarded this image as positive for leakage.

independently reviewed all of the 256 axial, $1 \mathrm{~mm}$ thick, slices for each patient to determine whether the GBCA leakage around the cortical veins was positive or negative. The number of arachnoid granulations was counted as positive for high signal intensity in nodular structures with a diameter of $1.5 \mathrm{~mm}$ or greater and protruding into the SSS. Any discrepancies between the two observers were discussed and a consensus was obtained.

\section{Statistical analysis}

A Mann-Whitney $U$ test was used to compare the average age between the negative and the positive group. The receiver operating characteristic (ROC) curve analysis for the Youden index (sensitivity + specificity -1 ) maximization was used to set the age cut-off value for the prediction of GBCA leakage around the cortical veins. A kappa test was used to evaluate the agreement between the two observers regarding 
the GBCA leakage. A Mann-Whitney $U$ test was used to compare the average number of arachnoid granulations between the negative and the positive group. The ROC curve analysis for the Youden index maximization was used to set the number of arachnoid granulations cut-off value for the prediction of GBCA leakage around the cortical veins. For the evaluation of the number of arachnoid granulations protruding into the superior sagittal sinus, a Pearson's correlation coefficient and an intraclass correlation coefficient (ICC) between the two observers were calculated. We used $5 \%$ as a threshold to determine statistical significance. The software $\mathrm{R}$ (version 3.3.2, R Foundation for Statistical Computing, Vienna, Austria, https://www.R-project.org/) was used for all statistical analyses.

The ethics committee of our institution approved this retrospective study with a waiver of consent from the patients.

\section{Results}

The GBCA leakage into the subarachnoid space around the cortical veins was negative in 35 patients and positive in 155 patients. In the negative group, the average age was $33 \pm 11$ years, and the average number of arachnoid granulations was $1.2 \pm 1.4$. In the positive group, the average age was $55 \pm 12$ years, and the number of arachnoid granulations was $1.5 \pm 1.1$. There were significant differences in both age and number of arachnoid granulations between the negative and positive groups $(P<0.01)$ (Figs. 3 and 4$)$.

Regarding the leakage of the GBCA around the cortical veins between the two observers, discrepancies were seen in four patients ( $\kappa$-value of $0.9315,95 \%$ confidence level: $0.865-0.998)$ and the number of arachnoid granulations was different by one in two patients (Pearson's correlation coefficient of $0.996,95 \%$ confidence level: $0.995-0.997$, ICC of 0.996).

In the ROC analysis for the age and leakage of the GBCA around the cortical veins, an area under the curve (AUC) of 0.905 (95\% confidence level: 0.848-0.963) was calculated. The Youden index reached a maximum at 37.317 years (sensitivity of 0.942 and specificity of 0.771 ; Fig. 5).

For the number of arachnoid granulations and the leakage of the GBCA around the cortical veins, the threshold was 1.000 with an AUC of 0.657 (95\% confidence level: $0.542-0.772$ ), at the Youden index maximum (sensitivity of 0.923 and specificity of 0.429 ; Fig. 6).

\section{Discussion}

Although there was no strict age threshold as previously reported, ${ }^{1}$ the age of 37 years is correct as a threshold for these imaging parameters of 3D-real IR at $4 \mathrm{~h}$ after intravenous injection of a single dose of GBCA (IV-SD-GBCA).

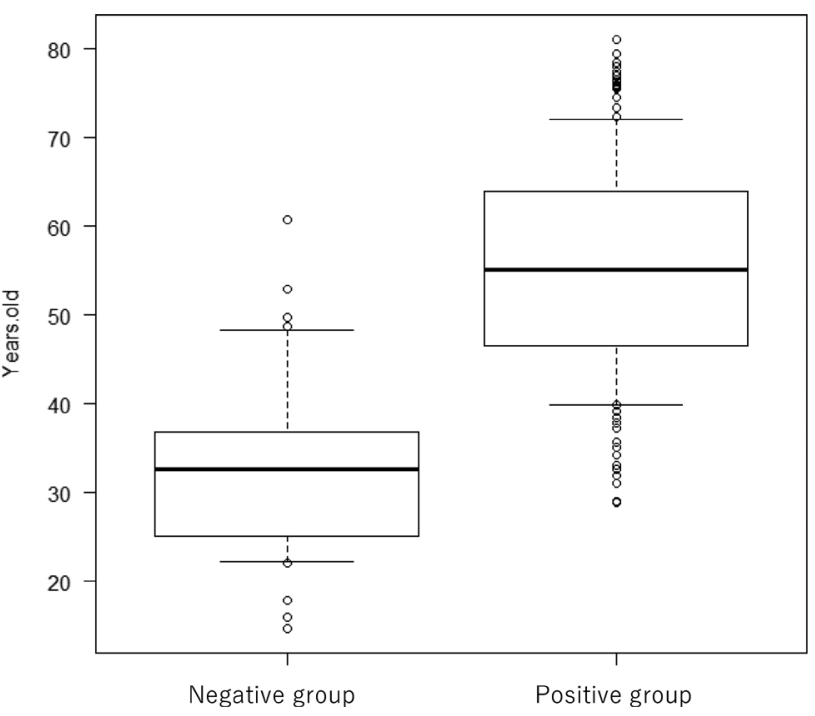

Fig. 3 A box-and-whisker plot of the age distribution of the negative group $(n=35)$ versus the positive group $(n=155)$ for leakage of the gadolinium contrast agent into the subarachnoid space around the cortical veins. The lower side of the rectangle is the first quartile (25th percentile value) and the upper side is the 75th percentile value. The thick horizontal line in the rectangle is the median. The horizontal line under the whisker indicates the 10th percentile value, and the horizontal line above the whisker indicates the 90th percentile value. There is overlap between the two groups, however the average of each group differs significantly $(P<0.01)$.

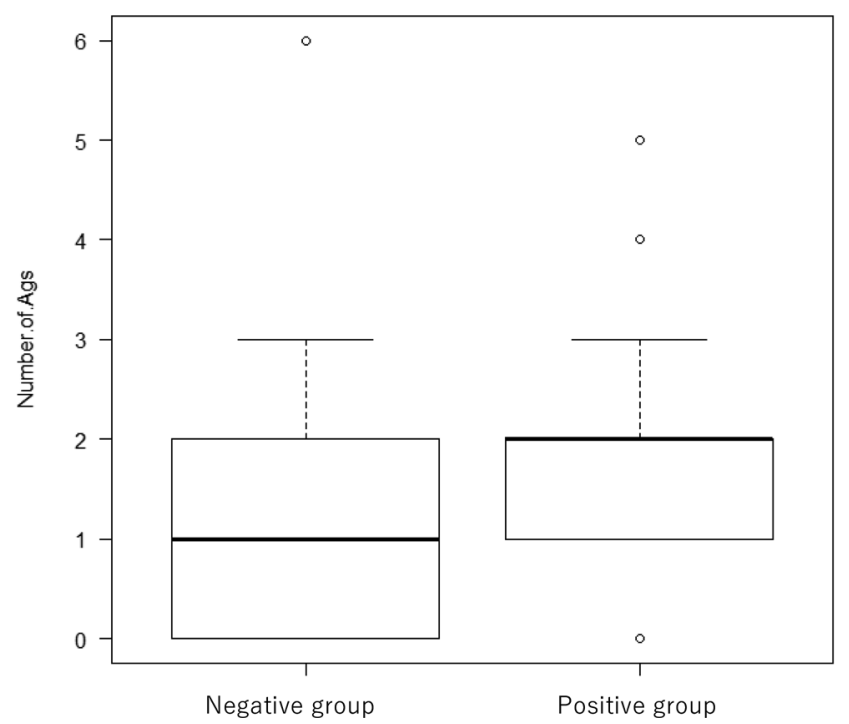

Fig. $4 \mathrm{~A}$ box-and-whisker plot of the number of arachnoid granulations protruding into the superior sagittal sinus of the negative group $(n=35)$ versus the positive group $(n=155)$ for the leakage of the gadolinium contrast agent into the subarachnoid space around the cortical veins. The lower side of the rectangle is the first quartile (25th percentile value) and the upper side is the 75th percentile value. The thick horizontal line in the rectangle is the median. The horizontal line under the whisker indicates the 10th percentile value, and the horizontal line above the whisker indicates the 90th percentile value. Although the average of the groups differs significantly ( $P$ $<0.01$ ), the overlap is large. Ags = arachnoid granulations. 


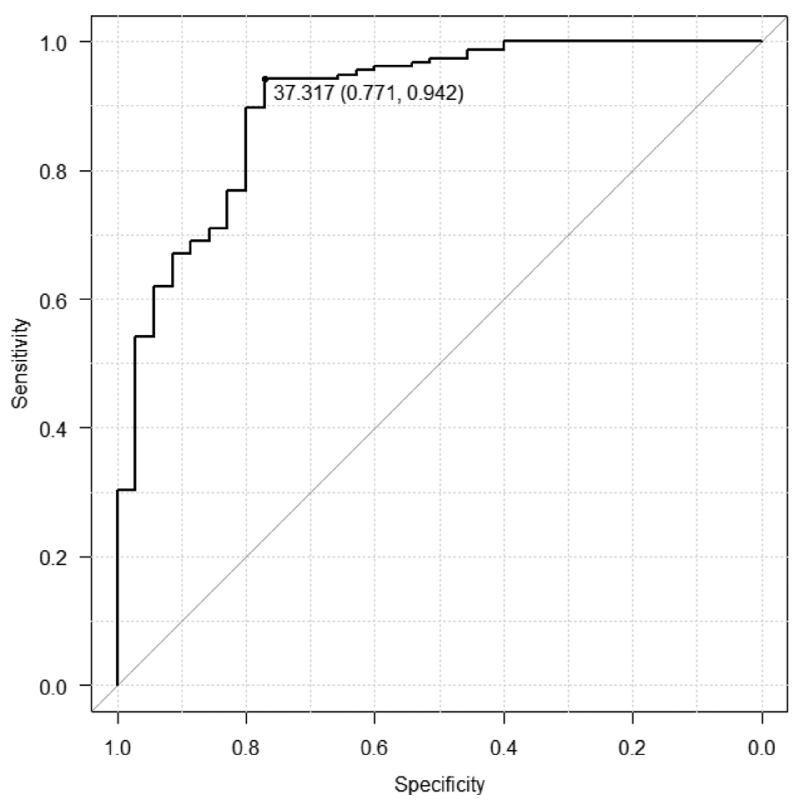

Fig. 5 The graph shows the result of the receiver operating characteristic (ROC) curve analysis of the GBCA leakage into the subarachnoid space around the cortical veins and the patient age. The Youden index is maximized at an age cut-off of 37.317 years (sensitivity of 0.942 and specificity of 0.771 ). The AUC is 0.905 (95\% confidence interval: 0.848, 0.963). GBCA, gadolinium-based contrast agent; AUC, area under the ROC curve. Youden index = sensitivity + specificity -1 .



Fig. 6 The graph shows the result of the receiver operating characteristic (ROC) curve analysis of the GBCA leakage to the subarachnoid space around the cortical veins and the number of arachnoid granulations. The Youden index is maximized at the number of arachnoid granulations' cut-off of 1.000 (sensitivity of 0.923 and specificity of 0.429 ). The AUC is 0.657 (95\% confidence interval: $0.542,0.772)$. GBCA, gadolinium-based contrast agent; AUC, area under the ROC curve. Youden index = sensitivity + specificity -1 .
The leakage of the GBCA around the cortical veins might be related to increased permeability of the blood-brain barrier $(\mathrm{BBB})$ and congestion of the meningeal lymphatic channels. ${ }^{11,12}$ In addition, since it is estimated that the function of the glymphatic system is also affected, it may become an important index in the future for the study of neurodegenerative diseases such as Alzheimer's disease. ${ }^{15,17}$ It is an important first step to identify an age threshold as shown in this study.

An experimental study using regular FLAIR imaging, which temporarily opened the blood-brain barrier with focused ultrasound (FUS) showed that the amount of GBCA leakage into the subarachnoid space after intravenous injection was increased by FUS. ${ }^{18}$

According to the previous report, the leakage around the cortical veins after intravenous injection of GBCA is limited to the perivascular space around the cortical veins at $5 \mathrm{~min}$ after intravenous injection, after which it spreads into the surrounding the subarachnoid space. ${ }^{1}$ Furthermore, images in which the perivascular space around the cortical veins is directly connected to the meningeal lymphatic vessels near the SSS have been shown. ${ }^{19}$ If the permeability of the pialsheath $^{20,21}$ encapsulating the cortical veins remains intact even with age, the results of the present study suggest that there might be congestion due to stenosis in the vicinity of the junction with the meningeal or meningeal lymphatics with age. ${ }^{11,12}$ Increased permeability of the BBB, resulting in an increase in the amount of the GBCA flowing into the perivascular space around the veins ${ }^{18}$ may lead to an increase in the leakage of the GBCA around the cortical veins. It has been reported previously that aging increases BBB permeability ${ }^{22}$ and also decreases the meningeal lymphatic vessel diameter and coverage. ${ }^{17}$

Although the number of arachnoid granulations and the leakage of GBCA around the cortical vein seem to be slightly related, as seen in the boxplot in Fig. 4, the overlap between the positive and negative groups is quite large. Due to a lower AUC value (0.657) from the number of arachnoid granulations (Fig. 6), it might not be appropriate to use this metric to predict leakage for an individual diagnosis. The results of the present study might have been affected by the fact that there was no arachnoid granulation, which was large enough to inhibit venous sinus flow. ${ }^{23}$

It is speculated that the deposition of the GBCA in the brain after the repeated intravenous administrations could be related to the GBCA concentration in cerebrospinal fluid (CSF). ${ }^{8,23-25}$ Increased GBCA concentration in CSF due to aging has also been reported. ${ }^{24}$ The results of the present study are consistent with these previous investigations.

Steroid has the effect of reducing inflammation and permeability of disrupted BBB ${ }^{26}$ In the patients of the present study, no patients received systemic steroid therapy within 2 months from MR examination.

This study has some limitations. All patients were imaged under suspicion of endolymphatic hydrops and were 
not completely healthy. For the imaging method used in the present study, we did not confirm the linearity of the gadolinium concentration in CSF, nor the detection rate of positive findings for leakage. We relied on subjective evaluation, although a signal intensity threshold was used. The number of patients in the negative group $(n=35)$ was much smaller than the number of patients in the positive group $(n=155)$. We did not obtain the super-delayed images more than $4 \mathrm{~h}$ after IV-SD-GBCA. Further research would be warranted to know the peak leakage timing for each age.

There are several applications for leakage of GBCA into the subarachnoid space around the cortical veins that should be investigated in the future. These include investigations for Alzheimer's disease and normal pressure hydrocephalus, as well as the effects of circadian rhythm, arteriosclerosis, and other physiological parameters such as gender or blood glucose levels.

\section{Conclusion}

Intravenously administered GBCA leaks into the subarachnoid space around the cortical veins in most patients over 37 years of age. However, it should be noted that it can be found occasionally in patients under 37 years of age. The age of 37 is not a perfect cut-off for predicting GBCA leakage into the subarachnoid space around the cortical veins. However it may be an adequate cut-off value in most cases. The number of arachnoid granulations protruding into the superior sagittal sinus is not a good predictor of GBCA leakage.

\section{Acknowledgment}

This study was supported in part by JSPS KAKENHI Grant Numbers of 18K19510, and 17H04259.

\section{Conflicts of Interest}

The authors declare that they have no conflicts of interest.

\section{References}

1. Naganawa S, Nakane T, Kawai H, Taoka T. Age dependence of gadolinium leakage from the cortical veins into the cerebrospinal fluid assessed with whole brain 3D-real inversion recovery MR imaging. Magn Reson Med Sci 2019; 18:163-169.

2. Kato Y, Bokura K, Taoka T, Naganawa S. Increased signal intensity of low-concentration gadolinium contrast agent by longer repetition time in heavily $\mathrm{T}_{2}$-weighted-3DFLAIR. Jpn J Radiol 2019; 37:431-435.

3. Naganawa S, Kawai H, Taoka T, Sone M. Improved 3D-real inversion recovery: a robust imaging technique for endolymphatic hydrops after intravenous administration of gadolinium. Magn Reson Med Sci 2019; 18:105-108.

4. Naganawa S, Kawai H, Taoka T, Sone M. Improved HYDROPS: imaging of endolymphatic hydrops after intravenous administration of gadolinium. Magn Reson Med Sci 2017; 16:357-361.

5. Freeze WM, Ter Weele DN, Palm WM, et al. Optimal detection of subtle gadolinium leakage in CSF with heavily $\mathrm{T}_{2}$-weighted fluid-attenuated inversion recovery imaging. AJNR Am J Neuroradiol 2019; 40:1481-1483.

6. Naganawa S, Kawai H, Sone M, Nakashima T. Increased sensitivity to low concentration gadolinium contrast by optimized heavily T2-weighted 3D-FLAIR to visualize endolymphatic space. Magn Reson Med Sci 2010; 9:73-80.

7. Deike-Hofmann K, Reuter J, Haase R, et al. Glymphatic pathway of gadolinium-based contrast agents through the brain: overlooked and misinterpreted. Invest Radiol 2019; 54:229-237.

8. Taoka T, Naganawa S. Glymphatic imaging using MRI. J Magn Reson Imaging 2020; 51:11-24.

9. Louveau A, Smirnov I, Keyes TJ, et al. Structural and functional features of central nervous system lymphatic vessels. Nature 2015; 523:337-341.

10. Aspelund A, Antila S, Proulx ST, et al. A dural lymphatic vascular system that drains brain interstitial fluid and macromolecules. J Exp Med 2015; 212:991-999.

11. Zou W, Pu T, Feng W, et al. Blocking meningeal lymphatic drainage aggravates Parkinson's disease-like pathology in mice overexpressing mutated $\alpha$-synuclein. Transl Neurodegener 2019; 8:7.

12. Yanev P, Poinsatte K, Hominick D, et al. Impaired meningeal lymphatic vessel development worsens stroke outcome. J Cereb Blood Flow Metab 2019:271678X18822921.

13. Yang L, Kress BT, Weber HJ, et al. Evaluating glymphatic pathway function utilizing clinically relevant intrathecal infusion of CSF tracer. J Transl Med 2013; 11:107.

14. Iliff JJ, Lee H, Yu M, et al. Brain-wide pathway for waste clearance captured by contrast-enhanced MRI. J Clin Invest 2013; 123:1299-1309.

15. Rasmussen MK, Mestre $H$, Nedergaard M. The glymphatic pathway in neurological disorders. Lancet Neurol 2018; 17:1016-1024.

16. Lenck S, Radovanovic I, Nicholson P, Hodaie M, Krings T, Mendes-Pereira V. Idiopathic intracranial hypertension: the veno glymphatic connections. Neurology 2018; 91: 515-522.

17. Da Mesquita S, Louveau A, Vaccari A, et al. Functional aspects of meningeal lymphatics in ageing and Alzheimer's disease. Nature 2018; 560:185-191.

18. Meng $\mathrm{Y}$, Abrahao A, Heyn CC, et al. Glymphatics visualization after focused ultrasound-induced bloodbrain barrier opening in humans. Ann Neurol 2019; 86:975-980.

19. Naganawa S, Ito R, Taoka T, Yoshida T, Sone M. The space between the pial sheath and the cortical venous wall may connect to the meningeal lymphatics. Magn Reson Med Sci 2019.

20. Abbott NJ, Pizzo ME, Preston JE, Janigro D, Thorne RG. The role of brain barriers in fluid movement in the CNS: is there a 'glymphatic' system? Acta Neuropathol 2018; 135:387-407.

21. Pizzo ME, Wolak DJ, Kumar NN, et al. Intrathecal antibody distribution in the rat brain: surface diffusion, perivascular transport and osmotic enhancement of delivery. J Physiol (Lond) 2018; 596:445-475. 
22. Erickson MA, Banks WA. Age-associated changes in the immune system and blood-brain barrier functions. Int J Mol Sci 2019; 20:1632.

23. Nicholson P, Brinjikji W, Radovanovic I, et al. Venous sinus stenting for idiopathic intracranial hypertension: a systematic review and meta-analysis. J Neurointerv Surg 2019; 11:380-385.

24. Nehra AK, McDonald RJ, Bluhm AM, et al. Accumulation of gadolinium in human cerebrospinal fluid after gadobutrol- enhanced MR imaging: a prospective observational cohort study. Radiology 2018; 288:416-423.

25. Taoka T, Naganawa S. Gadolinium-based contrast media, cerebrospinal fluid and the glymphatic system: possible mechanisms for the deposition of gadolinium in the brain. Magn Reson Med Sci 2018; 17:111-119.

26. Thal SC, Neuhaus W. The blood-brain barrier as a target in traumatic brain injury treatment. Arch Med Res 2014; 45:698-710. 